\title{
ORIGINAL ARTICLE Perceived adherence barriers among patients failing second-line antiretroviral therapy in Khayelitsha, South Africa
}

\author{
W Barnett, ${ }^{1}$ MPH; G Patten, ${ }^{2}$ MSc; B Kerschberger, ${ }^{2}$ MD, MPH; K Conradie, ${ }^{2}$ MB ChB, DCH (SA), DipHIVMan (SA); \\ D B Garone, ${ }^{2} \mathrm{MB} \mathrm{ChB}, \mathrm{MD}$; G van Cutsem, ${ }^{2,3} \mathrm{MD}$, DTM\&H, MPH; C J Colvin, ${ }^{3} \mathrm{PhD}$ (Anthropology), MPH \\ ${ }^{1}$ School of Public Health and Family Medicine, University of Cape Town, South Africa \\ ${ }^{2}$ Médecins Sans Frontières, Khayelitsha, Cape Town, South Africa \\ ${ }^{3}$ Centre for Infectious Disease Epidemiology and Research, School of Public Health and Family Medicine, \\ University of Cape Town, South Africa
}

Corresponding author: G Patten (gpatten@gmail.com)

Background. The recent scale-up of antiretroviral therapy (ART) coverage in resource-limited settings has greatly improved access to treatment. However, increasing numbers of patients are failing first- and second-line ART.

Objective. To examine factors affecting adherence to second-line ART from the perspective of clinic staff and patients, assessing both individual and structural perceived barriers.

Methods. Research was conducted at a large primary care tuberculosis (TB)/HIV clinic in Khayelitsha, a peri-urban township in Cape Town, South Africa. Participants were drawn from a Médecins Sans Frontières-run programme to support patients failing second-line ART. A qualitative research approach was used, combining multiple methodologies including key informant interviews with staff $(n=11)$, in-depth interviews with patients $(n=10)$ and a Photovoice workshop $(n=11)$. Responses and photographs were coded by content; data were transformed into variables and analysed accordingly.

Results. Staff identified drinking, non-disclosure, not using condoms and pill fatigue as barriers to ART adherence, while patients identified side-effects, not using condoms and a lack of understanding concerning medication timing. With respect to service delivery, staff identified a need for continued counselling and educational support following ART initiation. Patients were concerned about missing medical records and poor staff attitudes in the clinic.

Conclusion. These findings identify discrepancies between provider and patient perceptions of barriers to, and facilitators of adherence, as well as of service delivery solutions. This highlights the need for on-going counselling and education following ART initiation, improved quality of counselling, and improved methods to identify and address specific barriers concerning medication adherence.

S Afr J HIV Med 2013;14(4):170-176. DOI:10.7196/SAJHIVMED.981

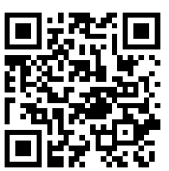

In 2010, the World Health Organization estimated that 34 million people were living with HIV/AIDS (PLWHA) - of whom more than 30 million were living in low- and middle-income countries. ${ }^{[1]}$ Around 9.7 million were receiving antiretroviral therapy (ART) in 2012, more than a 30-fold increase in ten years. ${ }^{[2]}$ With an increased number of people receiving ART, first- and second-line treatment failure has become more common. ${ }^{[1,3]}$

Treatment failure results in increased morbidity and mortality for PLWHA, with larger numbers requiring more expensive second- and third-line treatment, and an increased risk of HIV transmission, including drug-resistant strains..$^{[3]}$ For ART to be effective, adherence rates must be between $90 \%{ }^{[4,5]}$ and $95 \%{ }^{[6,7]}$ Studies also indicate that many failures are the result of suboptimal adherence, rather than due to the development of antiretroviral (ARV) resistance, suggesting a potential for continued efficacy with improved adherence, especially on protease inhibitor (PI)-based regimens. ${ }^{[8-10]}$
Patients failing second-line regimens have no further treatment options available in the public sector in sub-Saharan Africa, as third-line regimens are too costly. ${ }^{[11]}$ There is an escalating need to mitigate failure rates and improve the effectiveness of ART within existing public health structures. ${ }^{[12]}$

We sought to investigate: (i) major barriers to, and facilitators of ART adherence in this population; and (ii) areas of ART service delivery that shape patient behaviour. We examined the experiences of patients who failed both first- and second-line ART, identified through routine viral load (VL) testing. The most commonly cited barriers to adherence included: side-effects; regimen complexity; sociocultural factors such as complex dosing schedules, alcohol and substance abuse; economic factors; and patient-provider relationships. ${ }^{[13-17]}$ No studies specifically addressing barriers to, or facilitators of adherence in patients with multiple episodes of treatment failure and re-adherence were identified. The majority of patients in this study achieved successful re-suppression following second-line ART failure. Further 
work is needed to better understand the successful re-suppression of HIV in these patients. ${ }^{[8]}$

\section{Methods \\ Setting}

This study was conducted at Ubuntu Clinic, a public sector primary care HIV/TB clinic in Khayelitsha, a peri-urban township in Cape Town, South Africa (SA). Khayelitsha, with more than 500000 residents, has one of the highest burdens of HIV infection nationally and worldwide, with an antenatal HIV prevalence of $>26 \%$ in 2010. ${ }^{[18]}$ ARVs have been available since 2001 and currently there are more than 20000 PLWHA in the area receiving ART. ${ }^{[19]}$ Ubuntu Clinic serves the biggest and oldest cohort of ART patients in Khayelitsha. By the end of 2011, 6296 patients were receiving ART, of whom 463 (7.4\%) were receiving second-line treatment. Currently, 20 new patients per month are starting secondline ART. ${ }^{[18]}$

Treatment success is assessed through VL testing. Provincial guidelines recommend that a VL test is performed at four and 12 months after treatment initiation and on a yearly basis thereafter. ${ }^{[11]}$ After an initial high VL (defined as $>1000$ copies $/ \mathrm{ml}$ ), patients attend adherence counselling and a follow-up VL is taken three months thereafter. Two consecutives VLs $>1000$ copies $/ \mathrm{ml}$ confirm virological failure, after which patients should be switched to a second-line ART regimen. At Ubuntu Clinic, after five years of receiving ART, an estimated $14 \%$ of patients experienced virological failure and $12 \%$ were switched to a second-line regimen. ${ }^{[20,21]}$ Previous studies have found second-line failure rates in SA to be as high as $33 \%{ }^{[22]}$ and $40 \%,{ }^{[25]}$ respectively. The latter study conducted viral genotyping at Ubuntu Clinic and found that only two of 37 second-line failure patients had developed drug resistance. ${ }^{[23]}$

Médecins Sans Frontières (MSF) is an international non-governmental organisation that has worked closely with the Western Cape Department of Health during the scale-up of ART coverage in Khayelitsha. In 2010, MSF, in collaboration with Ubuntu Clinic staff, developed and implemented a patient-centred model of care for patients failing secondline ART. The programme provides a dedicated space, and individual and group counselling support. At the time of this study (August 2011), 49 patients were enrolled in the second-line failure clinic. This population allowed a first look at a small, but growing group of patients with multiple treatment failures in a setting with one of the highest HIV burdens worldwide.

\section{Study population}

Patients in MSF's second-line failure programme (patients with at least one VL $>1000$ copies $/ \mathrm{ml}$ ) were approached to participate in the study. Of the 49 patients meeting these criteria, 12 were included based on availability and willingness to participate. Nurses, counsellors and doctors were selected from both the MSF programme and Ubuntu Clinic staff as key informants; they were purposively sampled to include those with the most exposure to patients receiving second-line ART.

\section{Data collection}

This study used multiple methods including in-depth patient and key informant interviews and a Photovoice workshop. ${ }^{[2]}$ Semi-structured interviews were developed with input from all co-investigators on the project. Key informant interviews focused on staff perspectives regarding reasons for treatment failure and barriers in healthcare delivery. Patient interviews focused on individual reasons for treatment failure and perceptions of clinic service delivery.
Patients also engaged in a Photovoice workshop, a participatory qualitative research method where participants are given disposable cameras to take photographs of predetermined themes, and then engage with each other around the meanings and experiences behind the photographs. ${ }^{[24]}$ Participants took part in two day-long workshops. The first introduced Photovoice and, with the participants, developed themes to explore the relationship between treatment and the people, places and ideas in their daily lives. Participants were then given two weeks to take photographs and return the cameras to the research team for development. In the second workshop, participants chose three of their photographs to share, and researchers facilitated discussion and the identification of themes. Twelve patients consented to be in the study, all took part in the Photovoice workshop; however, two could not be found for in-depth interviews following multiple attempts. One had relocated from the area, and another could not be located.

\section{Data analysis}

Interviews were conducted in English $(n=3)$ and isiXhosa $(n=7)$, depending on participant preference, with the aid of a translator. Interviews and Photovoice sessions were tape-recorded and transcribed; each was conducted by one of two separate investigators. Interview content and photographs were analysed to identify recurring themes. The initial analysis was done by a single investigator, and the review of themes identified was performed by all co-investigators. A process of coding and categorisation of data content assisted in bringing meaning to the responses and photographs. ${ }^{[25,26]}$ Data were transformed into variables, with interviews and Photovoice data analysed separately.

\section{Ethical approval}

The study was approved by the Human Research Ethics Committee and Institutional Review Board of the Faculty of Health Sciences, University of Cape Town. Each participant gave written consent for their involvement in the study, including consent to publication of their photographs.

\section{Results \\ Sample characteristics}

Of the 10 patients interviewed, nine were female and one was male. All lived in Khayelitsha. The patients received first-line ART for a mean of 32 months (range 13 - 63) and second-line ART for a mean of 38 months (range 10 - 72). After being switched to second-line ART, patients experienced their first elevated VL ( $>1000$ copies $/ \mathrm{ml}$ ) at a mean of 12 months (range 4 - 41). Of the 11 key informant interviews, three were nurses, four were counsellors and four were doctors. Staff had worked a mean of 66 months (range 6 - 168) in the clinic.

\section{Barriers and enablers to adherence Patient-cited barriers}

No patient responded that a single barrier caused their treatment failure and the majority $(n=6)$ stated that they had made an active decision to stop taking their medication due to the barriers identified. The top reasons for ART failure (each cited by 3 patients) were side-effects, not using condoms, a lack of understanding around medication timing, didanosine (DDI) time delay between medication and food intake, and large pill size (Table 1).

\section{Key-informant-cited barriers}

The main adherence barriers cited by staff were patient drinking $(n=9)$, non-disclosure $(n=8)$, not using condoms $(n=6)$, pill fatigue $(n=5)$ and forgetting to take medication $(n=5)$ (Table 1$)$. 
Table 1. Barriers to ART adherence cited patients and key informants

\begin{tabular}{|c|c|c|}
\hline \multirow[b]{2}{*}{ Issue } & \multicolumn{2}{|c|}{ Barriers, $n(\%)$} \\
\hline & $\begin{array}{l}\text { Key-informant-cited } \\
(n=11)\end{array}$ & $\begin{array}{l}\text { Patient-cited } \\
(n=10)\end{array}$ \\
\hline Drinking & $9(82)$ & $1(10)$ \\
\hline Disclosure & $8(73)$ & $1(10)$ \\
\hline Not condomising & $6(55)$ & $3(30)$ \\
\hline Pill fatigue & $5(45)$ & $1(10)$ \\
\hline Forgetting & $5(45)$ & $2(20)$ \\
\hline Patients not honest with clinic staff & $3(27)$ & - \\
\hline Stigma & $3(27)$ & - \\
\hline Side-effects & $3(27)$ & $3(30)$ \\
\hline Lack of food in the home & $2(18)$ & - \\
\hline Life stress & $2(18)$ & $2(20)$ \\
\hline Insufficient support & $2(18)$ & - \\
\hline Treatment partner not working & $1(9)$ & - \\
\hline Didanosine/one-hour delay ${ }^{*}$ & $1(9)$ & $3(30)$ \\
\hline Denial & $1(9)$ & - \\
\hline Feeling better & $1(9)$ & - \\
\hline Embarrassed about defaulting & $1(9)$ & - \\
\hline Timing of medication & $1(9)$ & $3(30)$ \\
\hline Staff shouting & $1(9)$ & - \\
\hline Gave up & - & $1(10)$ \\
\hline Has not accepted HIV status & - & $1(10)$ \\
\hline Unable to keep appointment & - & $1(10)$ \\
\hline Pills too large & - & $3(30)$ \\
\hline
\end{tabular}

\section{Side-effects}

One-third of patients identified side-effects as a reason for treatment failure, citing nausea, vomiting, stomach pains and cramping: 'I have this diarrhoea in my stomach and it's cramping ... it started my viral to go up and up and up because I skip now because I'm scared that maybe I'm going to the church and my stomach maybe want to run.' (P06)

'Patients are not taking three of the tablets, taking two because one has got side-effects.' (KI02)

Another patient on second-line ART stopped because she did not realise her VL would go up quickly and was surprised when it became detectable. One-third of key informants also identified side-effects as a barrier.

\section{Not condomising}

One-third of patients blamed their failure on not using condoms: 'I had a boyfriend and didn't condomise with the boyfriend. After that I ... failed and was changed to secondline.' (P04)

Not using condoms also emerged as a central theme in the key informant interviews, with six of 11 respondents citing it as a reason for non-adherence. Key informants did appear to understand this risk to treatment success as a consequence of transmission of resistant strains. However, they seemed to inflate the risk of this occurring. One key informant described a tendency of staff to defer to an 'easy' explanation for treatment failure.

\section{Timing of medication}

At the time of ART initiation, counsellors instructed patients to take their pills twice daily at exactly the same time in the morning and evening. This is laid out as one of the 'rules' of ART adherence and is presented in a concrete format, allowing very little scope for the patients when times clashed with their schedules: 'To keep the time is too difficult. I take my pills at 7 but sometimes wake up at 8 and the time has passed.' (P05)

One patient (P04) stated that she often missed doses because of her work schedule.
Another blamed his counsellor for not telling him to take his medication twice daily, e.g. at 07 h00 and 19h00. One-third of patients indicated that they had defaulted on first-line ART due to such strict parameters: 'Before they said to us, if you used to take your tablets at 8 o'clock in the morning or night you can't take it at 9 o'clock because it's too late. But [MSF counsellors] said it's not late, you must take the tablets. If you forget, maybe it's two hours or one hour, you can take your tablets.' (P02)

Many patients cited this increased latitude around timing as helpful to becoming readherent, allowing them more freedom to adjust timing around their schedules. Only one key informant mentioned timing of medication as a barrier to patient adherence.

\section{DDI time delay}

Until 2009, SA guidelines recommended DDI as part of its second-line ART regimen. This required patients to take the medication one hour before eating. This time-delay was identified by one-third of patients and one key informant as a reason for treatment failure.

'You must leave [medication] for the hour and then you forget to take other tablets after that. You take it maybe at 6 o'clock; you have to run at 7 oclock for the train ... You think okay that time is past, so you have to drink at night. That's why your viral load is so grown.' (P06)

'We picked up that they were having the problem with the DDI delay and forgetting.' (KI01)

\section{Pills too large}

One-third of patients stated that they had difficulty taking their medication because of the large pill size. Two of these patients discontinued taking their second-line regimen for this reason: 'It was getting so difficult to take second-line. It was a big pill and I decided to stop.' (P04)

Pill size was not noted in the key informant interviews as a reason for non-adherence.

\section{Patient drinking}

One patient identified drinking as a factor in her treatment failure. In the key informant interviews, however, alcohol use emerged as the most cited reason for failure (cited by 9 ). Staff said that this affected adherence in two ways: Firstly, patients often forget to take their medication when drinking. Secondly, staff reported that, as with the timing of medication, patients understood that they could not drink and take their ARVs literally and stopped treatment altogether: '[The patient] sees that he 
is much better and he will start to drink again. It's whereby most of them are failing because when they are drinking, they don't take their medication, they stop their medication.' (KI02)

\section{Non-disclosure}

One patient identified non-disclosure as a barrier, citing the difficulty of maintaining ART without support at home where she felt she had to hide her medication: 'Some of them they don't tell their partners that they are positive and are on treatment. They need to disclose to friends, to partners.' (KI10)

Eight of the 11 key informants indicated that non-disclosure was problematic. Many of their patients felt the need to hide their medication and would often not take it when travelling or if others were present at work or at home.

\section{Pill fatigue}

One patient identified pill fatigue as a reason for defaulting, noting: 'I just keep getting tired sometimes. To take treatment everyday is not nice.' (P03); 'Second-line patients are more likely to have problems because of pill fatigue.' (KI09)

Five of the 11 key informants identified pill fatigue as a barrier.

\section{Forgetting}

Five of the 11 key informants identified patients forgetting to take their pills as a barrier. This was often cited with various reasons, ranging from patients being busy with work or family obligations, travelling without medication, and the lack of a plan for treatment adherence (alarm or friend/family to remind them).

Two of 10 patients identified forgetting as a barrier.

\section{Service delivery barriers \\ Patient-cited clinic obstacles}

Interviews with patients revealed difficulties with healthcare delivery at Ubuntu Clinic, with some patients citing missing medical records $(n=6)$ and clinic staff shouting at patients $(n=6)$. Few, however, cited these problems in response to questions on defaulting or suboptimal adherence. Rather, many seemed to view them as an expected part of clinic attendance; two patients stated that clinic problems affected their adherence or their attendance at the clinic: 'Sometimes they will shout you if you ask something ... shouting at the top of their voices. You feel not happy and you go home and feel unhappy. And next time you say I'm not going to this clinic anymore.' (P02)

\section{Key-informant-cited clinic obstacles}

Staff identified a lack of continued counselling support following ART initiation $(n=8)$ and insufficient education for patients $(n=3)$ as key obstacles. One counsellor noted: 'The point where we are failing is to really find out exactly why [the patient] is failing and try to fix the thing that makes them to fail first-line ... there is no time to focus on the problem, instead we are just providing the medication without support.' (KI09)

Staff also highlighted the need for increased follow-up to catch adherence issues earlier on. One clinician identified counselling as a critical but under-utilised component: 'There is so much pressure for roll-out of getting more patients onto ARVs, getting nurses dishing out ARVs ... counsellors are being overlooked but they are a critical part of the whole process.' (KI08)

Three of the 11 key informants responded that there was no need for improvement and seven did not think that the clinic needed more time or resources for patients. These staff felt that patients should engage better with the healthcare system, that many obstacles faced by patients are difficult for the clinic to solve, or that current resources could be managed better.

\section{Patient perspectives on the MSF programme}

Patients cited feeling more comfortable and free to share problems $(n=7)$, shorter wait times $(n=6)$, seeing the same staff $(n=4)$ and support groups $(n=2)$ as reasons why they preferred MSF's programme to the larger clinic: 'I feel free now that on this side of the clinic. I can share everything ... when I come here I feel at home because before when I was taking medicine on that side [larger clinic], I was afraid whether I had done right or done wrong.' (P04)

A more patient-centred environment enabled more open discussion of barriers to adherence and facilitated the resolution of issues.

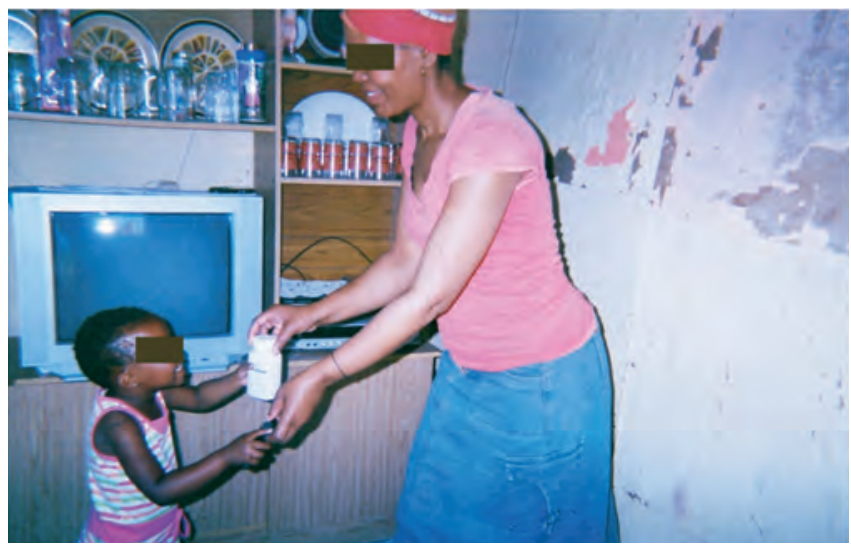

Fig. 1. The daughter of a patient reminds her mother to take her medication.

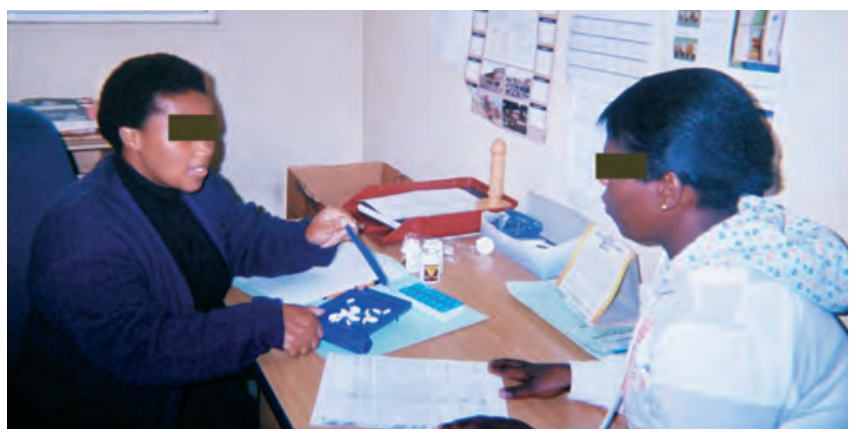

Fig. 2. A counsellor explaining medication parameters to a patient.

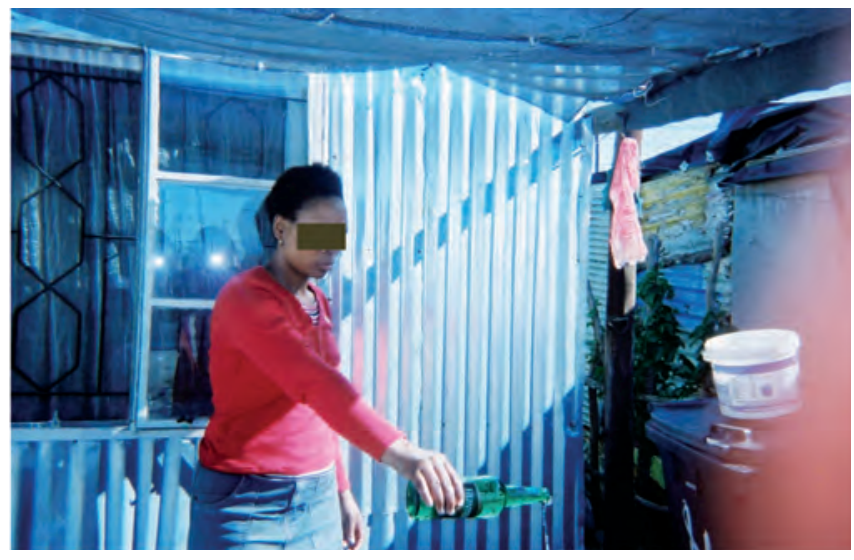

Fig. 3. A patient who struggled with an alcohol abuse problem depicts herself pouring out the remaining alcohol in her home. 


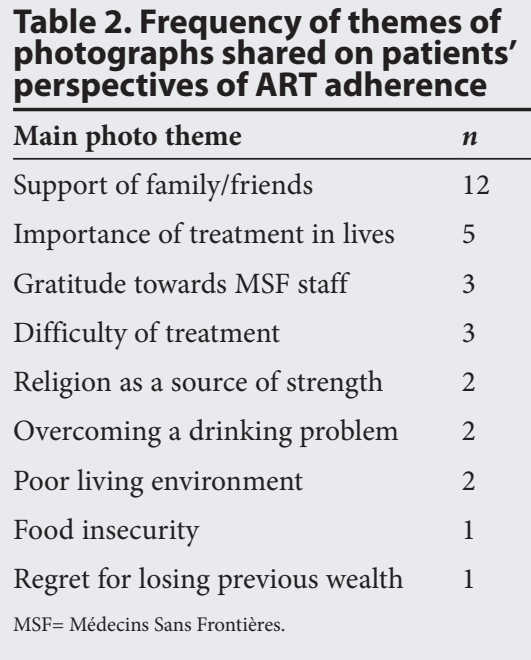

\section{Photovoice patient perspectives}

The Photovoice component of the study elicited a different patient perspective on treatment compared with the interviews. The photographs and the workshop discussion became a platform for sharing successes and sources of strength. Each patient chose three pictures to share (Table 2). Of 33 total photographs shared, only nine illustrated negative aspects of patients' lives, such as poor living conditions, difficulty remembering treatment, poor clinic service and not having family support. Yet, when presented by patients, these negative aspects were often treated as barriers overcome or obstacles to get past.

The remaining photographs (24/33) showed supportive family and friends ( $n=11$ ) (Fig. 1), the importance of treatment $(n=5)$, gratitude towards the MSF clinic staff ( $n=3$ ) (Fig. 2), religion as a source of strength $(n=2)$, and overcoming drinking problems $(n=2)$ (Fig. 3). There was a notable difference in the themes presented by patients as part of the Photovoice analysis and those that emerged in the interviews. This is likely due to the difference in methodology and format. Photovoice involved individual presentation to the full group; patients seemed to experience this format as a type of support group with a focus on sharing positive experiences, often to encourage others in the group by describing facilitators to adherence and sources of support: 'I believe in tablets, because it's my life and I will be taking treatment for life. Not for me, but for the kids too.' (P04)

\section{Discussion}

South Africa's National Strategic Plan on HIV, STIs and TB: 2012 - 2016 calls for $80 \%$ of PLWHA to be receiving ART by 2016, ${ }^{[27]}$ expanding from the $56 \%$ of those eligible who were receiving ART in 2009. ${ }^{[28]}$ However, this focus on initiating patients on treatment has largely ignored the rising numbers of patients failing treatment. ${ }^{[1,29]}$ We aimed to identify barriers and facilitators to long-term ART adherence in the context of second-line treatment failure from the perspective of staff and patients.

Interview themes most frequently identified as barriers to treatment adherence differed between patients and staff. Staff identified drinking, non-disclosure, not using condoms, pill fatigue and forgetting to take medication as barriers to adherence, while patients identified side-effects, not using condoms and a lack of understanding around medication timing. With respect to service delivery, staff identified a need for continued counselling and educational support following ART initiation. Patients were concerned about missing medical records and poor staff attitudes in the clinic.

Patients as well as staff had a tendency to blame treatment failure on factors that were external to themselves and their role in treatment adherence. This is consistent with previous findings in a similar setting, where ART patients who adhered tended to ascribe this to internal strength, whereas when they failed, external factors were identified. ${ }^{[30]}$ In the current study, the principal patient-identified barriers were sideeffects, not condomising, DDI, large pills and not understanding medication - all but condomising point toward obstacles external to the patient; whereas, staff identified patient drinking, not disclosing, not condomising and pill fatigue most frequently - all of which focus more on patient behaviour.

In the principal obstacles identified, the only one common to both patients and staff was not using a condom. When staff were questioned further on this topic, it became clear that they seemed to understand not condomising as contributing to the spread of resistant HIV strains within the population. However, given the small numbers of those failing secondline ART, the likelihood was very low of any patients in this sample contracting a resistant strain rather than failure resulting from nonadherence. Given the complexities involved in identifying behaviours that contribute to non-adherence, one explanation is that both patients and staff found not condomising a factor that is easily identifiable and relatively easily addressed, causing both to overemphasise its role. This over-emphasis adds to the knowledge gap patients experience in understanding the reasons for treatment failure, reducing their ability to address and identify actual adherence issues.

For some patients, ART failure resulted from a lack of understanding around the parameters of their medication. For others, the issues were psychosocial in nature, involving bad living environments or a lack of support at home. Yet, some patients made an active decision to stop taking medication and became re-adherent when issues such as sideeffects, pill size or time delay were addressed.

Patients enrolled in the second-line failure clinic receive patient-centred support with individual and group counselling. Unpublished data show a high VL re-suppression rate among those enrolled in the programme. This is likely due to improved access to staff, continuity of staff and more supportive clinic environment, allowing for quicker follow-up and more open discussion of issues affecting adherence. This is consistent with findings from similar studies where strong and open relationships between patients and healthcare providers facilitate adherence. ${ }^{[30]}$

There is currently an information gap between patients experiencing difficulties with treatment and clinic staff addressing those issues. There are personal and structural reasons for this, stemming from patients not informing staff when experiencing difficulties, not understanding which behaviours will lead to raised VLs and not feeling comfortable with staff. The most patient-cited clinic obstacle was staff shouting at patients; however, some patients also highlighted the benefit of feeling open and able to share their issues with the MSF programme staff. This serves to emphasise the importance of trust and communication between patients and staff, particularly between patients and counsellors; patients' difficulties with treatment remained unresolved until the clinic environment changed, allowing for open discussion and management of those issues. Though previous research has shown similar barriers to adherence including poor patient-staff relationships, untimely addressing of adherence issues and inadequate counselling following initiation of ART, ${ }^{[31]}$ the patient population in this study showed that improving these issues within service delivery can lead to improved adherence and VL suppression.

Many staff responded that there was no need for an improvement in service delivery and that the clinic had sufficient resources 
to serve its patient population, highlighting a tendency among some staff not to be critical of the status quo or look for methods to improve current service delivery. The present focus on increasing ART coverage and the relatively few patients who are failing treatment has concentrated efforts to increase the number of patients receiving ART, leaving little time to focus on the increasing rate of ART failure.

\section{Study limitations}

Interviews conducted in isiXhosa were translated during the interview in real time by MSF staff, which may have influenced patient response, either limiting patient openness due to the presence of clinic staff or limiting the interviewer interaction because of the language barrier. The high proportion of female respondents limited the generalisability of the study findings, suggesting that our results may only be appropriate to a female population failing second-line ART. Though the proportion of females attending the second-line ART clinic at the time was $68 \%$, participation of males was low due to difficult schedules and willingness to participate. Only one male participated in the study, making it difficult to determine whether responses were gender-biased. The study population comprised a small group of patients who had a very specific context of care delivery within a programme separate from standard government clinical care. Thus, their experiences within a more adequately resourced environment than public clinical care is certain to have affected their perspectives on service delivery as well as their high level of re-adherence. Moreover, because of the relatively low numbers of patients failing second-line ART, the large bulk of staff experience is with patients receiving first-line ART. As the number of patients failing second-line ART increases, so will staff experience with these patients, offering a future opportunity to assess staff perspectives on barriers to, and facilitators of adherence.

\section{Study significance}

This study focused on a patient population that included among the first patients in SA who have experienced virological failure on secondline ART medication. Yet, many of these patients have been able to suppress their VLs within the MSF-run programme. This success highlights the importance of both counsellors and clinicians providing the necessary patient support - to ensure that patients are adequately equipped to initiate ART successfully and to serve as the front line for identifying problems as they arise. Many of the patients who became readherent benefitted from open relationships with clinic staff, enabling discussion and management of adherence issues. Prioritising patientstaff relationships and strengthening support mechanisms to identify adherence issues early on offers an opportunity for the current system of ART initiation and support to improve patient outcomes. The largely positive themes elicited by Photovoice illustrate that many multiplefailure patients feel positively about their treatment and lives and, with improved support mechanisms, this can translate into improved adherence and outcomes. Although some patients did make an active decision to stop taking ART, all continued to attend the clinic and engage with the healthcare system, demonstrating an opportunity for clinic staff to reduce failure rates in a patient population with no treatment option following failure of a second-line ART regimen.

\section{Recommendations}

The frequency with which not using condoms was cited as a factor contributing to ART failure should be examined further at the staff and patient level to ensure that messaging is not misleading and that patients address all behaviours affecting adherence. Continued followup, especially with regards to easily altered behaviour or misunderstood medication parameters (e.g. timing, missed doses, forgetting) should be explored to identify how best to equip patients for ART regimens. Continuity of care and patients' comfort with staff is another area that could be explored to identify whether and how that may have contributed to improved patient outcomes in this group. Though few patients were found to have resistant HIV strains following genotyping at Ubuntu Clinic, the incidence of primary or secondary infection with resistant strains should be explored. This is likely to become an increasingly important aspect of ART failure as the prevalence of resistant strains increases.

Acknowledgements. We acknowledge the dedicated staff at Ubuntu Clinic and the participants who were involved in this study. The data and findings from this study will be made available upon request.

\section{References}

1. World Health Organization. Antiretroviral Therapy. http://www.who.int/hiv/ topics/treatment/en/index.html (accessed 26 May 2012).

2. World Health Organization. Global Update on HIV Treatment 2013: Results, Impact and Opportunities. http://apps.who.int/iris/ bitstream/10665/85326/1/9789241505734_eng.pdf (accessed 7 November 2013).

3. World Health Organization. Towards Universal Access: Scaling Up Priority HIV/AIDS Interventions in the Health Sector. http://www.who.int/hiv/ pub/2010progressreport/en/ (accessed 26 May 2012).

4. Sethi AK. Adherence and HIV drug resistance. HIV Clin Trials 2004;5:112-115.

5. Arnsten JH, Demas PA, Farzadegan H, et al. Antiretroviral therapy adherence and viral suppression in HIV-infected drug users: Comparison of self-report and electronic monitoring. Clin Infect Dis 2001;33:1417-1423. [http://dx.doi. org/10.1086/323201]

6. Catz SL, Kelly JA, Bogart LM, et al. Patterns, correlates, and barriers to medication adherence among persons prescribed new treatments for HIV disease. Health Psychol 2000;19:124-133.

7. Paterson DL, Swindells S, Mohr J, et al. Adherence to protease inhibitor therapy and outcomes in patients with HIV infection. Ann Intern Med 2000;133:21-30.

8. Levison JH, Orrell C, Gallien S, et al. Virologic failure of protease inhibitor-based second-line antiretroviral therapy without resistance in a large HIV treatment program in South Africa. PLoS ONE 2012;7:e32144. [http://dx.doi.org/10.1371/ journal.pone.0032144]

9. Ajose O, Mookerjee S, Mills EJ, et al. Treatment outcomes of patients on second-line antiretroviral therapy in resource-limited settings: A systematic review and meta-analysis. AIDS 2012;26(8):929-938 [http://dx.doi.org/10.1097/ QAD.0b013e328351f5b2]

10. Conradie K, Garone D, Patten G, et al. Intensive Adherence Counseling of Patients on ARV Treatment: A Model of Care for Patients Failing Second-line Treatment in Khayelitsha, South Africa. MSF UK Scientific Day, London, 2012.

11. National Department of Health. Clinical Guidelines for the Management of HIV \& AIDS in Adults and Adolescents. Pretoria: NDoH, 2011.

12. World Health Organization. Progress Report 2011: Global HIV/AIDS Response. http://www.who.int/hiv/pub/progress_report2011/en/index.html (accessed 24 May 2012).

13. Chesney M. Adherence to HAART regimens. AIDS Patient Care STDs 2003;17:169177. [http://dx.doi.org/10.1089/108729103321619773]

14. Hill Z, Kendall C, Fernandez M. Patterns of adherence to antiretrovirals: Why adherence has no simple measure. AIDS Patient Care STDs 2003;17:519-525. [http://dx.doi.org/10.1089/108729103322494311]

15. Remien RH, Hirky AE, Johnson MO, et al. Adherence to medication treatment: A qualitative study of facilitators and barriers among a diverse sample of HIV+ men and women in four US cities. AIDS Behav 2003;7:61-72.

16. Reynolds NR. Adherence to antiretroviral therapies: State of the science. Curr HIV Res 2004;2:207-214.

17. Sahay S K, Reddy S, Dhayarkar S. Optimizing adherence to antiretroviral therapy. Indian J Med Res 2011;134(6):835-849. [http://dx.doi.org/10.4103/0971-5916.92629]

18. Médecins Sans Frontières. 10 Years of HIV/TB care at Primary Health Care Level. Cape Town: MSF, 2011.

19. City of Cape Town Health Services. Health Information and Data. Cape Town: City of Cape Town, 2011.

20. Boulle A, Van Cutsem G, Hilderbrand K, et al. Seven-year experience of a primary care antiretroviral treatment programme in Khayelitsha, South Africa. AIDS 2010;24:563. [http://dx.doi.org/10.1097/QAD.0b013e328333bfb7]

21. Médecins Sans Frontières. MSF Report: Khayelitsha 2001 - 2011. http://www.msf. org.za/system/files/publication/documents/MSF_report_Khayelitsha_2001-2011. pdf?download=1 (accessed 20 February 2012). 
22. El-Khatib Z, Ekstrom AM, Ledwaba J, et al. Viremia and drug resistance among HIV-1 patients on antiretroviral treatment: A cross-sectional study in Soweto, South Africa. AIDS 2010;24:1679-1687. [http://dx.doi.org/10.1097/ QAD.0b013e32833a097b

23. van Zyl GU, van Mens TE, McIlleron $\mathrm{H}$, et al. Low lopinavir plasma or hair concentrations explain second-line protease inhibitor failures in a resource-limited setting. J Acquir Immune Defic Syndr 2011;56:333-339. [http://dx.doi.org/10.1097/ QAI.0b013e31820dc0cc]

24. Wang C, Burris MA. Photovoice: Concept, methodology, and use for participatory needs assessment. Health Educ Behav 1997;24:369-387.

25. Pope C, Mays N. Qualitative research: Reaching the parts other methods canno reach: An introduction to qualitative methods in health and health services research. BMJ Open 1995;311:42-45.

26. Elo S, Kyngäs H. The qualitative content analysis process. J Adv Nurs 2008;62:107 115. [http://dx.doi.org/10.1111/j.1365-2648.2007.04569.x]
27. South African National AIDS Council. National Strategic Plan on HIV, STIs and TB: 2012 - 2016. http://www.doh.gov.za/docs/stratdocs/2012/NSPfull.pdf (accessed 1 June 2012).

28. US Agency for International Development. USAID South Africa HIV/AID Profile. http://transition.usaid.gov/our_work/global_health/aids/Countries/africa/ southafrica.html (accessed 20 July 2012).

29. Cornell M, Grimsrud A, Fairall L, et al. Temporal changes in programme outcomes among adult patients initiating antiretroviral therapy across South Africa, 2002 - 2007. AIDS 2010;24:2263-2270. [http://dx.doi.org/10.1097] QAD.0b013e32833d45c5]

30. Sharada P, Wasti, Simkhada P, et al. Barriers to and facilitators of antiretroviral therapy adherence in Nepal: A qualitative study. J Health Popul Nutr 2012;30:410 419.

31. Sanjobo N, Frich JC, Fretheim A Barriers and facilitators to patients' adherence to antiretroviral treatment in Zambia: A qualitative study. Sahara J 2008;5:136-143. 